\title{
Sensors Based on Metal Nanoclusters Stabilized on Designed Proteins
}

\author{
Antonio Aires ${ }^{1,+}\left(\mathbb{D}\right.$, Elena Lopez-Martinez ${ }^{1,+}\left(\mathbb{D}\right.$ and Aitziber L. Cortajarena ${ }^{1,2, *}$ \\ 1 CIC biomaGUNE, Parque Tecnológico de San Sebastián, Paseo Miramón 182, 20014 San Sebastián, Spain; \\ aaires@cicbiomagune.es (A.A.); elopez@cicbiomagune.es (E.L.-M.) \\ 2 Ikerbasque, Basque Foundation for Science, Ma Díaz de Haro 3, 48013 Bilbao, Spain \\ * Correspondence: alcortajarena@cicbiomagune.es; Tel.: +34-943-00-5427 \\ + These authors contributed equally to this work.
}

Received: 2 October 2018; Accepted: 12 November 2018; Published: 15 November 2018

\begin{abstract}
Among all new nanomaterials, metal nanoclusters (NCs) have attracted special attention due to their interesting optical properties, among others. Metal NCs have been recently studied and used as sensors for different analytes. However, there is a need to explore the potential of these new sensors in a systematic manner and to develop new systems to broaden the possibilities that sensing offers to the industry. In this work, we show the potential use of repeat protein scaffolds as versatile templates for the synthesis and stabilization of various metal NCs, specifically $\mathrm{Au}, \mathrm{Ag}$, and CuNCs. The resulting protein-metal NCs hybrids are evaluated as sensors for different stimuli such as temperature, ions, or reactive oxygen species (ROS). Among the three protein-metal NCs, all performed nicely as temperature sensors, AuNCs responded to metal ions, and AgNCs were able to detect ROS.
\end{abstract}

Keywords: protein design; metal nanocluster; fluorescent probe; nanosensor; temperature sensor; metal sensors; ROS sensors

\section{Introduction}

Metal nanostructures are one of the most developed and studied systems in nanotechnology over the past decades, especially metal nanoparticles (NPs) and nanoclusters (NCs), due to their optical, magnetic, and catalytic properties. In particular, metal NCs smaller than $2 \mathrm{~nm}$ display different fluorescence emissions depending on their size, and also the molecules used for their stabilization [1]. The interesting optical properties that NCs feature are owed to their small size, comparable to Fermi wavelength of electrons, transforming the electrons from the performance of metal bulk into a quantum behavior, with discrete levels of energy. Fluorescent metal NCs have been widely used as sensors due to their response to certain changes in their medium such as temperature [2-7], oxidative stress, acidity, or the presence of different substances such as metal ions [8-10]. Metal NCs are usually synthetized using capping agents or proteins such as bovine serum albumin (BSA) as templates, although this approach does not give a precise control over the number of clusters per protein molecule or their position.

Protein engineering can be used for designing functionalized tools that fulfill the desired requirements. Simple modular proteins are excellent to use as templates for designing functional nanostructures because of their stability properties, modularity, and structure. For example, in a consensus tetratricopeptide repeat (CTPR), derived from natural tetratricopeptide repeat (TPR) sequences, only eight residues of the 34 within each CTPR unit are conserved and have structural significance, leaving plenty of space for mutations and changes alongside the protein. In the past, we showed an easy way to template AuNCs using CTPR proteins [11], and used this system to build a fluorescent probe for the recognition of a TPR-binding peptide. In the present work, we explore the 
repeat protein scaffolds as versatile templates for the synthesis and stabilization of different metal NCs and their potential use for sensing different stimuli, such as temperature, ions, and reactive oxygen species (ROS), with the main objective of developing an array modular sensing tools that could be combined in the same protein scaffold.

\section{Materials and Methods}

\subsection{Chemicals}

All chemicals were purchased from Sigma-Aldrich and used without further purification. Ultrapure reagent grade water (18.2 M $\Omega$, Wasserlab, Navarra, Spain) was used in all experiments.

\subsection{Purification of CTPR Protein}

The CTPR protein was produced following standard molecular biology protocols for recombinant protein expression. The CTPR3_cys protein cloned into pPro-EX-HTb vector were transformed in Escherichia coli C41 (DE3) cells. The cells were grown in Luria-Bertani media (LB) with ampicillin. The protein expression was induced by isopropyl $\beta$-D-1-thiogalactopyranoside (IPTG) at optical density of 0.6-0.8. After $16 \mathrm{~h}$ expression at $20^{\circ} \mathrm{C}$, cells were harvested, and the his-tagged proteins were purified via affinity chromatography using Ni-NTA resin. The his-tag was cleaved using Tobacco Etching Virus (TEV) protease, and a second Ni-NTA affinity column purification was performed in order to remove the his-tag and the TEV protease from the protein sample. The protein concentration was determined by absorbance at $280 \mathrm{~nm}$ using the extinction coefficient calculated from the amino acid composition. The CTPR3 with an additional cysteine residue at the C-terminal (C3_cys) has the following amino acid sequence:

\section{GAMDPGNSAEAWYNLGNAYYKQGDYDEAIEYYQKALELDPNNAEAWYNLGNAYYKQGDY DEAIEYYQKALELDPNNAEAWYNLGNAYYKQGDYDEAIEYYQKALELDPNNAEAKQNLGN AKQKQGC (Molecular weight: 14463.4 Daltons).}

\subsection{Synthesis and Characterization of Protein-Stabilized Metal Nanoclusters}

The protein-stabilized metal NCs were synthesized following a previously reported procedure using sodium ascorbate instead of ascorbic acid to provide the reducing environment [11]. Briefly, $1000 \mu \mathrm{L}$ of protein at $10 \mu \mathrm{M}$ were mixed with $\mathrm{HAuCl}_{4}, \mathrm{AgNO}_{3}$ or $\mathrm{CuSO}_{4}(50 \mu \mathrm{L} 10 \mathrm{mM}, 50$ equation respect to protein) for at least $30 \mathrm{~min}$ to allow the adsorption of metal ions to the protein's stabilizing sites. Then, the reduction of the metal ions to metal NCs was achieved by adding $50 \mu \mathrm{L}$ of sodium ascorbate at $100 \mathrm{mM}$ (10 equation respect to metal ions). The reaction was incubated at $37^{\circ} \mathrm{C}$ for $24 \mathrm{~h}$. The samples were concentrated to $500 \mu \mathrm{L}$ using Amicon ultrafiltration tubes with a 10-kDa membrane. Finally, the unreacted salts were removed by gel filtration chromatography, using an Illustra NAP-25 column equilibrated with phosphate-buffered saline (PBS). The purified samples were concentrated using Amicon $10 \mathrm{kDa}$ ultrafiltration tubes until $40 \mu \mathrm{M}$ and kept at $4{ }^{\circ} \mathrm{C}$ for further experiments. The samples were diluted in PBS when necessary for the experiments. The absorption and fluorescence spectra of protein stabilized metal NCs were recorded using a spectrophotometer UV-Vis (Jasco V630-Bio) and a fluorometer (Perkin Elmer LS55), respectively.

\subsection{Fluorescence Quantum Yield}

The fluorescence quantum yield $\left(\Phi_{\mathrm{x}}\right)$ was calculated using anthracene in ethanol as a reference $\left(\Phi_{\text {ref }}=0.27, \lambda_{\text {exc }}=370 \mathrm{~nm}\right.$ and $\left.\lambda_{\mathrm{em}}=423 \mathrm{~nm}\right)$ and the following formula:

$$
\phi_{\mathrm{x}}=\phi_{\mathrm{ref}} \frac{\operatorname{Grad}_{\mathrm{x}}}{\operatorname{Grad}_{\mathrm{ref}}}\left(\frac{\eta_{\mathrm{x}}^{2}}{\eta_{\mathrm{ref}^{2}}}\right)
$$


where $\operatorname{Grad}_{x}$ and $\mathrm{Grad}_{\mathrm{ref}}$ are the gradient from the plot of integrated fluorescence intensity versus absorbance at excitation wavelength, for the sample and the reference, respectively, and $\eta_{\mathrm{x}}$ and $\eta_{\text {ref }}$ are the refractive indexes of the solvents, water, and ethanol, respectively. Fluorescence measurements were performed using a fluorometer (Perkin Elmer LS55), and absorbance was recorded with a spectrophotometer UV-Vis (Jasco V630-Bio) in quartz cuvettes.

\subsection{Matrix Assisted Laser Desorption Ionization—Time Of Flight (MALDI-TOF) Mass Spectrometry}

Mass spectra were acquired on an Applied Biosystems Voyager Elite MALDI-TOF mass spectrometer with delayed extraction (Applied Biosystems, Framingham, MA, USA) equipped with a pulsed N2 laser $(\lambda=337 \mathrm{~nm})$. Sinapic acid was used as matrix. An extraction voltage of $20 \mathrm{kV}$ was used. All mass spectra were acquired in positive reflection mode using delayed extraction with an average of 50-100 laser shots. MALDI-TOF sample preparation included $1 \mu \mathrm{L}$ of the sample at $20 \mu \mathrm{M}$ mixed with $4 \mu \mathrm{L}$ of sinapic acid in 50:50 water/acetonitrile with $0.01 \%$ trifluoroacetic acid (TFA). Then, $1 \mu \mathrm{L}$ of the mixture was deposited onto the MALDI plate and allowed to air-dry. The instrument was externally calibrated using monoisotopic peaks from the sinapic acid matrix ( $\mathrm{MH}+$ at $m / z$ 225.071).

\subsection{Temperature Sensing}

The fluorescence spectra of a C3_cys-metal NCs suspension at $10 \mu \mathrm{M}$ of protein concentration were measured at different temperatures ranging from $25^{\circ} \mathrm{C}$ to $65^{\circ} \mathrm{C}$. The reversibility and the cycle stability of the metal NCs as temperature sensors were tested repeating the process for 5 cycles.

\subsection{Ion Detection}

To evaluate the selectivity of the protein-stabilized metal NCs towards several ion species, different ions at $10 \mu \mathrm{M}$ including $\mathrm{Na}^{+}, \mathrm{K}^{+}, \mathrm{Ag}^{+}, \mathrm{Ca}^{2+}, \mathrm{Ba}^{2+}, \mathrm{Cd}^{2+}, \mathrm{Co}^{2+}, \mathrm{Pb}^{2+}, \mathrm{Zn}^{2+}, \mathrm{Ni}^{2+}, \mathrm{Mn}^{2+}, \mathrm{Mg}^{2+}, \mathrm{Fe}^{2+}$, $\mathrm{Fe}^{3+}$, and $\mathrm{Hg}^{2+}$ were incubated with the metal NCs. Briefly, $500 \mu \mathrm{L}$ of the protein-stabilized metal NCs at $10 \mu \mathrm{M}$ were mixed with $5 \mu \mathrm{L}$ of the different ion solutions at $1 \mathrm{mM}$. After $30 \mathrm{~min}$ of reaction, $200 \mu \mathrm{L}$ of the reactant solution was transferred into a quartz cuvette for fluorescence spectra recording at room temperature.

Copper detection was evaluated by the incubation of protein-stabilized metal NCs in phosphate-buffered solution (10 mM phosphate $\mathrm{pH} 7.4)$ with ion solutions at different concentrations $(0-10 \mu \mathrm{M})$. Briefly, $5 \mu \mathrm{L}$ of $\mathrm{Cu}^{2+}$ solutions of different concentrations $(0-10 \mu \mathrm{M})$ obtained by serial dilution of the stock solution $(10 \mathrm{mM})$ were added to $500 \mu \mathrm{L}$ of the protein-stabilized metal NCs at $10 \mu \mathrm{M}$. After $30 \mathrm{~min}$ of incubation, $200 \mu \mathrm{L}$ of the solution were transferred into a quartz cuvette and the fluorescence spectra recorded at room temperature.

\subsection{ROS Detection}

For the detection of reactive oxygen species (ROS), the assays were performed using Rose Bengal as the synthesizer for ROS. This dye, when irradiated with green light, is able to produce singlet oxygen molecules. As a control for the presence of ROS species 2,2'-azino-bis(3-ethylbenzthiazoline-6-sulphonic acid) (ABTS) was used. 2,2'-azino-bis(3-ethylbenzthiazoline-6-sulphonic acid) is widely used in antioxidants studies as the reporter for oxidative stress environment [12,13]. In a typical experiment, $90 \mu \mathrm{L}$ of protein stabilized metal NCs at $40 \mu \mathrm{M}$ in phosphate-buffered solution were mixed with $10 \mu \mathrm{L}$ of Rose Bengal at $100 \mathrm{mM}$. The fluorescence was measured in a quartz cuvette before and after the irradiation with a green lamp during $15 \mathrm{~min}$ steps for a total time of $60 \mathrm{~min}$. In parallel, $25 \mu \mathrm{L}$ of ABTS were mixed with $10 \mu \mathrm{L}$ of Rose Bengal $(100 \mathrm{mM})$ and $65 \mu \mathrm{L}$ of phosphate-buffered solution as a positive control of ROS detection. Absorbance was measured using a Jasco spectrophotometer (model V630BIO UV-Vis) before and after irradiation with a green LED lamp during $15 \mathrm{~min}$ steps for a total time of $60 \mathrm{~min}$. 


\section{Results}

\subsection{Synthesis and Characterization of Protein-Stabilized Metal Nanoclusters}

Blue fluorescent protein stabilized metal NCs were synthesized in one step by reducing the metal salt $\left(\mathrm{HAuCl}_{4}, \mathrm{AgNO}_{3}\right.$ or $\left.\mathrm{CuSO}_{4}\right)$ with sodium ascorbate in the presence of $\mathrm{C} 3$ _cys protein at $37^{\circ} \mathrm{C}$ for $72 \mathrm{~h}$. The as-obtained protein stabilized metal NCs suspension are light brown under visible light (Figure 1A) and emit strong blue fluorescence under $365 \mathrm{~nm}$ irradiation (Figure 1B). The UV-visible spectra (Figure 1C) of the protein-stabilized metal compared with the spectrum of the protein, at the same concentration, showed in addition to the characteristic protein absorption at $280 \mathrm{~nm}$ the presence of small and broad peaks around 350-370 nm and in the case of AuNCs a small peak around $560 \mathrm{~nm}$ due to the presence of a small fraction of gold nanoparticles. The fluorescent protein-stabilized metal NCs showed maximum excitation and emission peaks at 375 and $453 \mathrm{~nm}$ (CuNCs), 371 and $445 \mathrm{~nm}$ (AgNCs), and 365 and $438 \mathrm{~nm}$ (AuNCs), respectively; while the protein without metal NCs did not produce any fluorescence signal (Figure 1D).
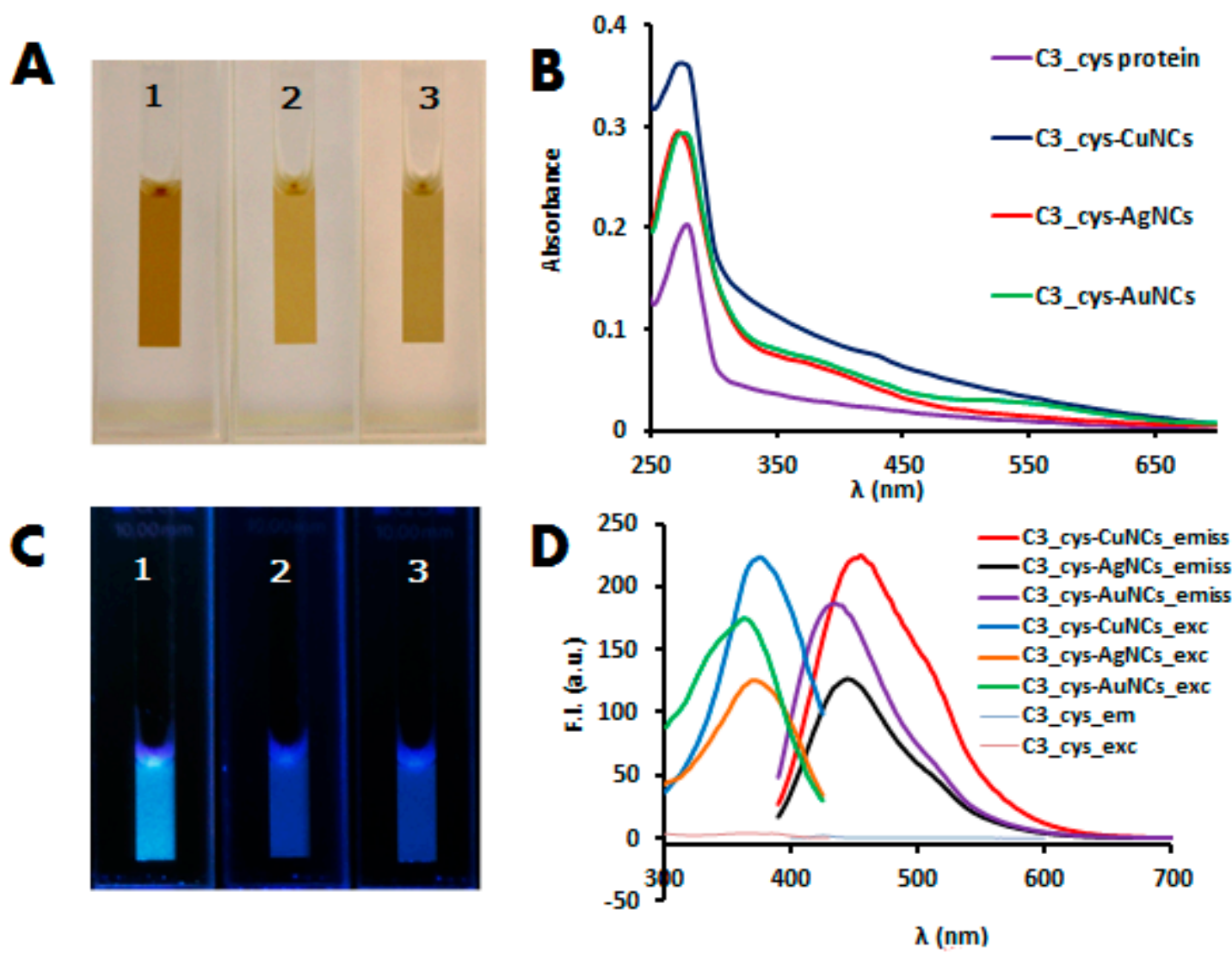

Figure 1. (A,C) images of consensus tetratricopeptide repeat (CTPR)-metal nanoclusters (NCs) solution under visible light and UV light: C3_cys-CuNCs (1), C3_cys-AgNCs (2), and C3_cys-AuNCs (3). (B) Absorption spectra of the non-conjugated CTPR protein and the synthetized protein-NCs conjugates. (D) Excitation and emission spectra of CTPR control protein without NCs and CTPR templated NCs.

The fluorescence quantum yield $\left(\Phi_{\mathrm{x}}\right)$ of $C 3$ _cys-CuNCs, C3_cys-AgNCs, and C3_cys-AuNCs were $4.1,2.6$, and $3.5 \%$, respectively, when anthracene was used as a reference. These values are in the same range as the ones reported for other protein-stabilized fluorescent metal NCs in the literature [14-16].

MALDI-TOF mass spectrometry was used to determine the size of the NCs. The protein-NCs complexes spectra showed clear shifts compared to the protein spectrum. However, the peaks were wider than the pure protein peak, indicating the presence of populations of protein with different numbers of metal atoms. Thus, it is possible that the laser irradiation during the MALDI-TOF 
acquisition was etching the NCs. The mass spectrum of C3_cys-CuNCs (Figure 2) showed a main peak at $m / z=14,693.34$ Da that corresponds to C3_cys-CuNCs with six copper atoms per protein (compared to a $m / z=14,321.65$ Da for free protein). Similarly, the mass spectrum of C3_cys-AuNCs showed a peak at $m / z=14,869.47$ Da indicating three gold atoms per protein, and the mass spectrum of C3_cys-AgNCs showed a peak at $m / z=14,789.94 \mathrm{Da}$ that corresponds to five silver atoms per protein. It is plausible that other NCs species were also formed; however, they were not clearly detected by MALDI-TOF mass spectrometry.

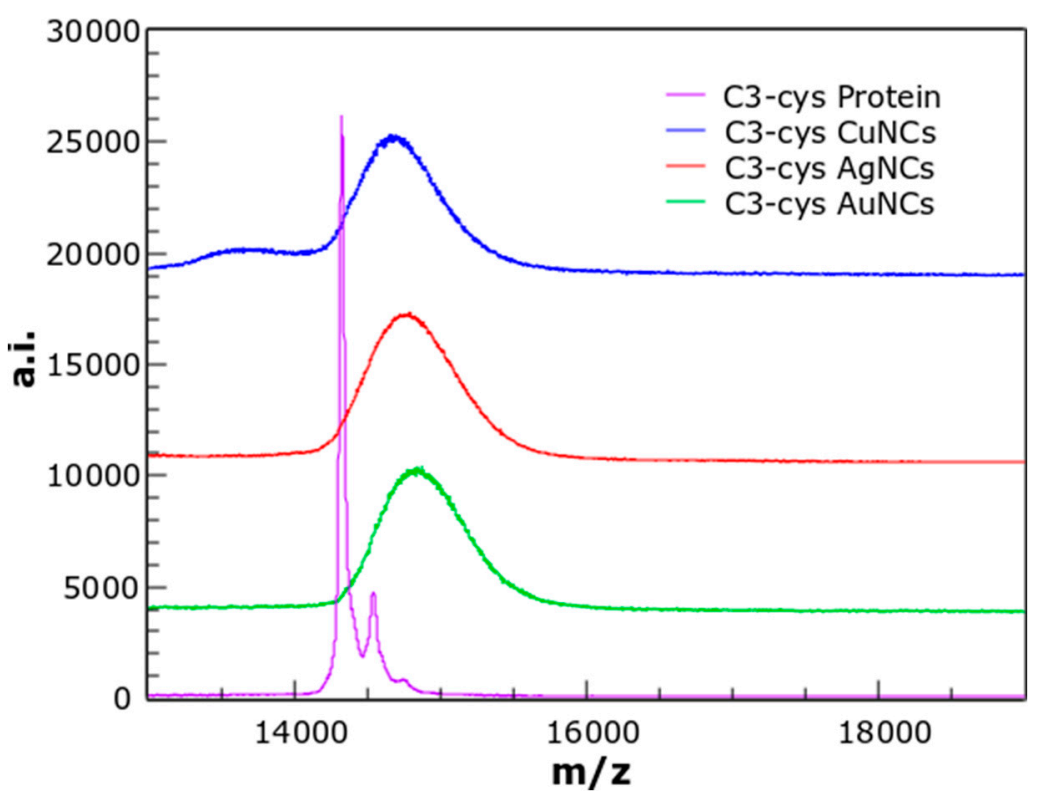

Figure 2. Mass spectra of non-conjugated C3_cys protein (magenta trace), and the protein-NCs conjugates: C3_cys-CuNCs (blue trace), C3_cys-AgNCs (red trace), and C3_cys-AuNCs (green trace). The shifts in the peaks relative to the protein spectrum correspond with the absorption of six $\mathrm{Cu}$ atoms, five Ag atoms, and three Au atoms.

\subsection{Temperature Sensing}

Protein stabilized metal NCs fluorescence was tested under a temperature range from $25{ }^{\circ} \mathrm{C}$ to $65{ }^{\circ} \mathrm{C}$ (Figure 3). The fluorescence decays up to $42.47 \%$ in the case of CuNCs, $45.84 \%$ for AgNCs, and $42.85 \%$ in the case of AuNCs (Figure 3E-G respectively). Comparatively, the effect of the temperature on the fluorescence of a control organic molecule, anthracene, is smaller (Figure 3D,H), showing a fluorescence quenching of $10.5 \%$ at the maximum temperature tested. This difference empowers the potential use of CTPR templated NCs as thermos-sensing devices due to their more linear response to temperature changes than some dyes as anthracene, although other organic dyes, such as Rhodamine C present also a linear response to temperature [17]. However, CTPR templated NCs present the advantage of being water-soluble and fluorescent in physiological conditions.

Potential hysteresis effects may hamper the use of a sensor as a reliable nanothermometer, thus, it is important to evaluate that the fluorescence intensity at a certain temperature is not dependent on the heating or cooling cycles and remains the same when the system is heating up or is cooling down. Hysteresis effects may emerge from local conformational changes of the protein at the surroundings of the metal NC [4]. Nevertheless, CTPR proteins present a good thermodynamic stability under the temperature range in which the sensor was evaluated [18,19] and a fully reversible thermal denaturation [20], therefore these scaffolds guarantee a system without hysteresis. 

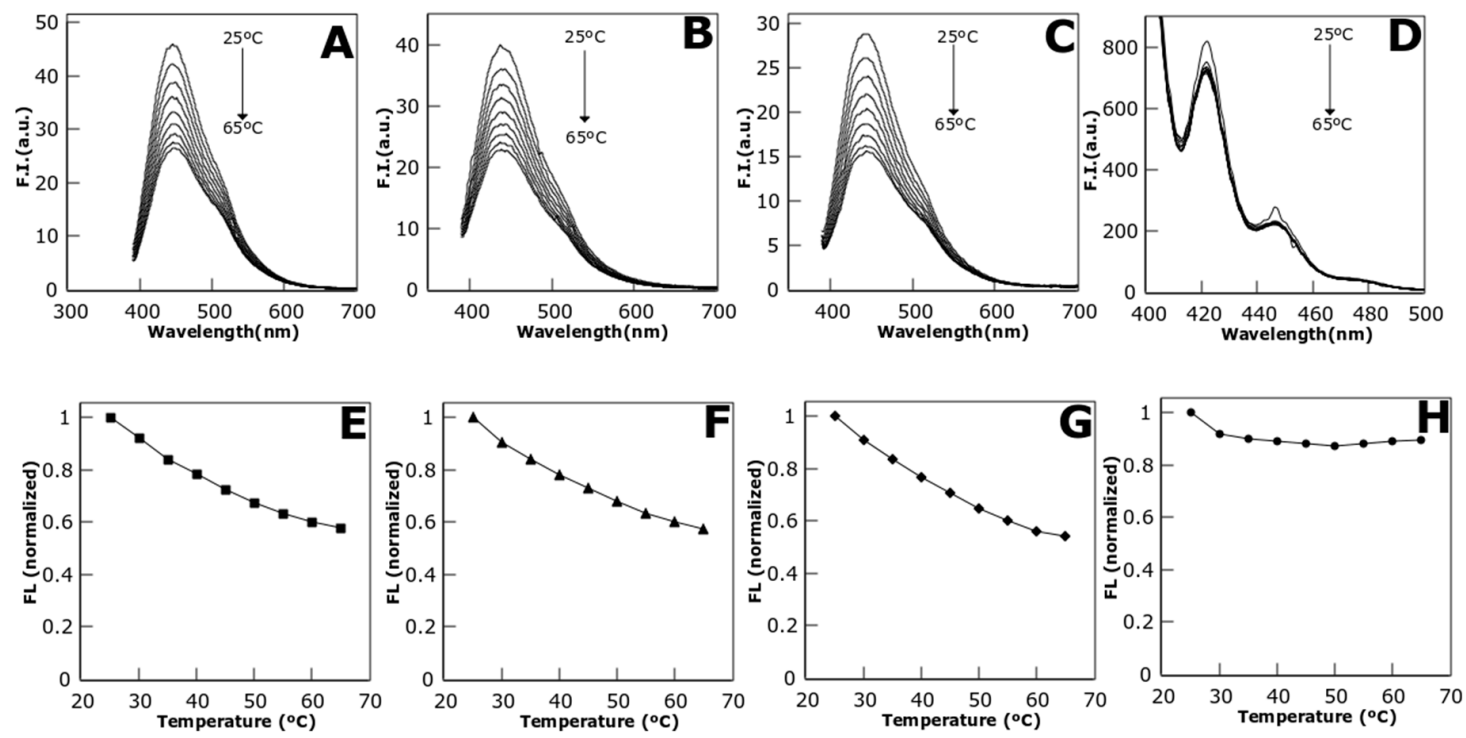

Figure 3. CTPR-templated metal NCs emission spectra under a temperature gradient from $25{ }^{\circ} \mathrm{C}$ to $65^{\circ} \mathrm{C}$ (upper row) for C3_cys-CuNCs (A); C3_cys-AgNCs (B); C3_cys-AuNCs (C); and anthracene, as reference molecule (D). Normalized fluorescence intensity vs. temperature (lower row) for C3_cys-CuNCs (E); C3_cys-AgNCs (F); C3_cys-AuNCs (G); and anthracene (H).

We also tested the stability of the fluorescent signal from the metal NCs through different cycles of temperature. We observe that the metal NCs can undergo several cycles of heating and cooling from $25^{\circ} \mathrm{C}$ to $65{ }^{\circ} \mathrm{C}$ without losing the temperature response and the resolution of the detection (Figure 4). The fact that CTPR-templated metal NCs can perform several cycles without losing fluorescence or temperature sensitivity makes them robust temperature sensors.
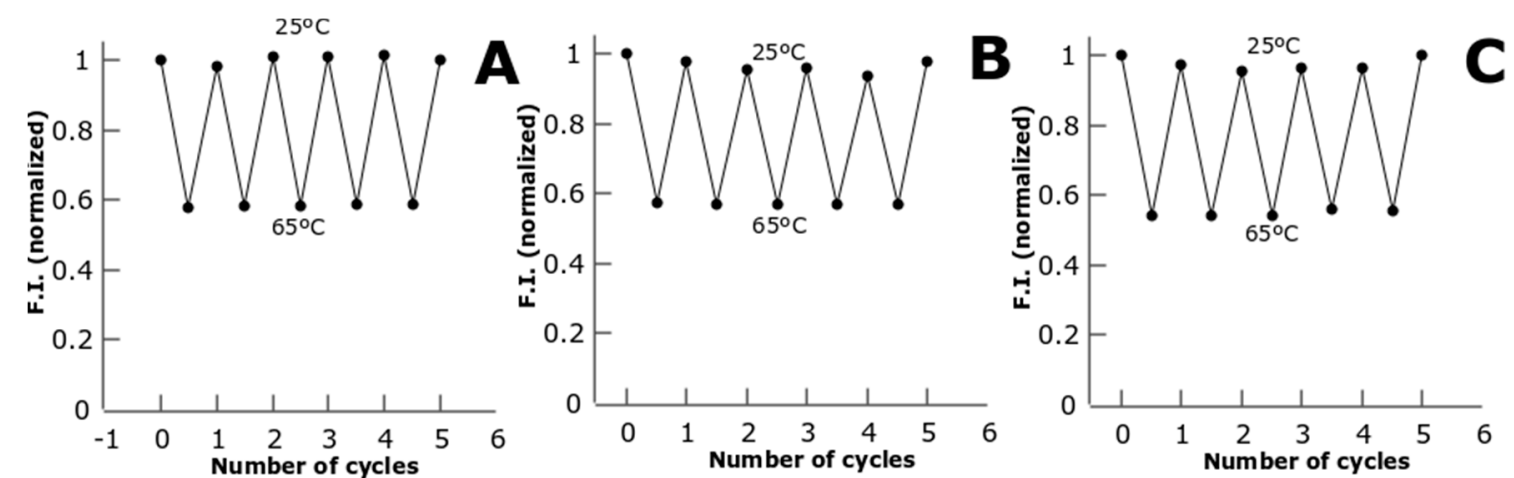

Figure 4. Normalized fluorescence intensity vs. the number of temperature cycles between $25^{\circ} \mathrm{C}$ and $65^{\circ} \mathrm{C}$ for C3_cys-CuNCs (A), C3_cys-AgNCs (B), and C3_cys-AuNCs (C).

\subsection{Ion Detection}

Several ions were tested as targets for the sensing properties of the protein-stabilized metal NCs. Among these ions, copper performed as the strongest quencher of the fluorescence emission of the metal NCs, for each of the NCs composition (Figure 5). Besides, AgNCs and AuNCs showed an enhanced fluorescence in response to the presence of $\mathrm{Fe}^{2+}$ and $\mathrm{Hg}^{2+}$, while this phenomenon is not observed for the CuNCs. Interestingly, other works have reported a quenching in fluorescence from AuNCs [21] and CuNCs [22] triggered by the presence of $\mathrm{Hg}^{2+}$ ions. 

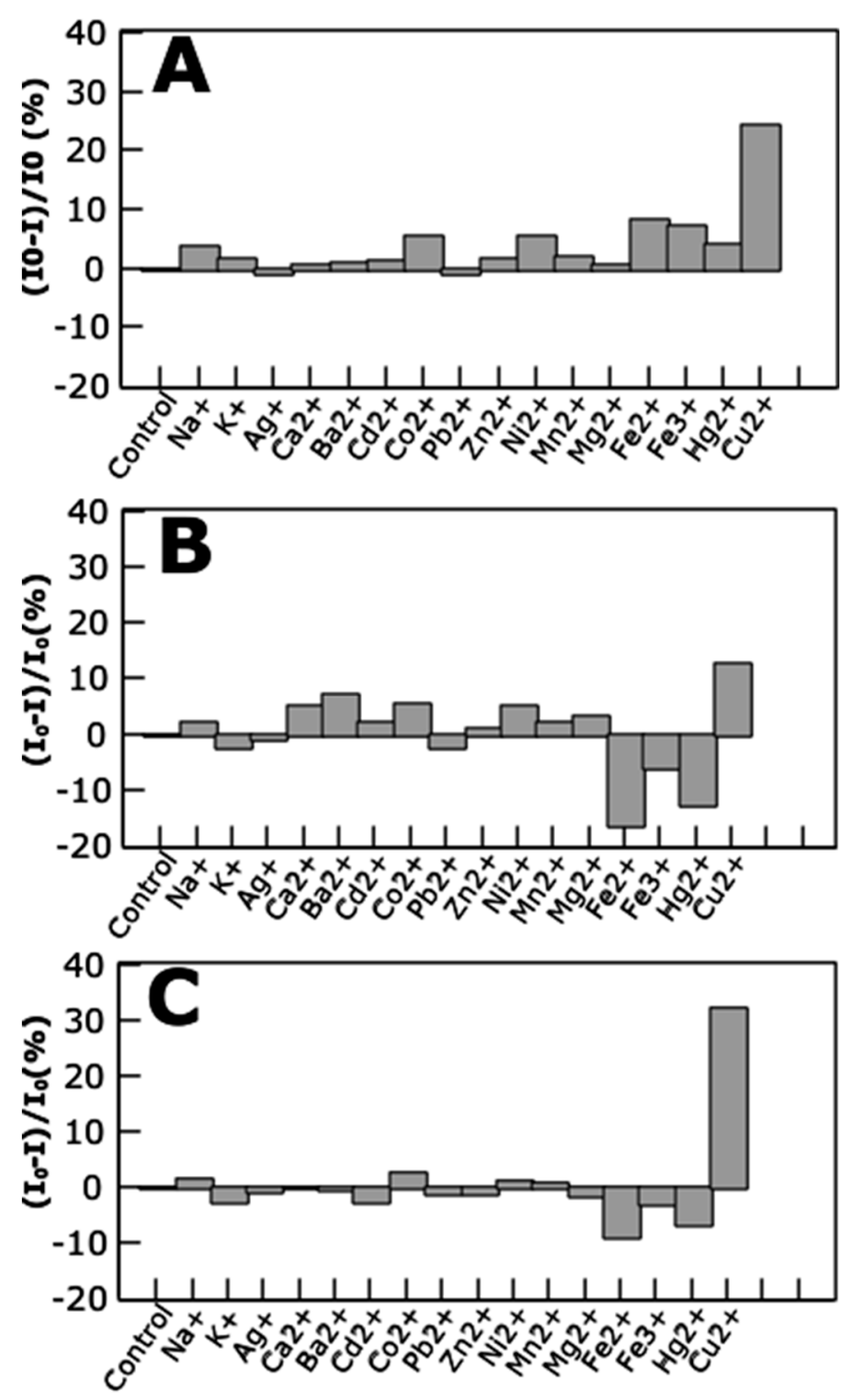

Figure 5. Ion detection by protein-stabilized NCs. The change in the fluorescence emission intensity of the protein-stabilized nanoclusters upon addition of different ions. C3_cys-CuNCs (A); C3_cys-AgNCs (B); and C3_cys-AuNCs (C).

Moreover, the protein-stabilized AuNCs were the most responsive to $\mathrm{Cu}^{2+}$ ions, being able to quench up to $20.4 \%$ of the fluorescence at $5 \mu \mathrm{M}$ of $\mathrm{Cu}^{2+}$ and $32.31 \%$ of the fluorescence at $10 \mu \mathrm{M}$ of $\mathrm{Cu}^{2+}$ (Figure 6). This phenomena has already been reported in previous works using BSA-AuNCs [8]; however, this work showed $\mathrm{Cu}^{2+}$ detection at $\mathrm{mM}$ concentration with only a $10 \%$ of fluorescence quenching at $10 \mu \mathrm{M} \mathrm{Cu}^{2+}$; whereas the CTPR templated AuNCs were sensitive to nanomolar concentrations $\mathrm{Cu}^{2+}$ and showed at $10 \mu \mathrm{M} \mathrm{Cu}^{2+}$ a quenching three times stronger. 

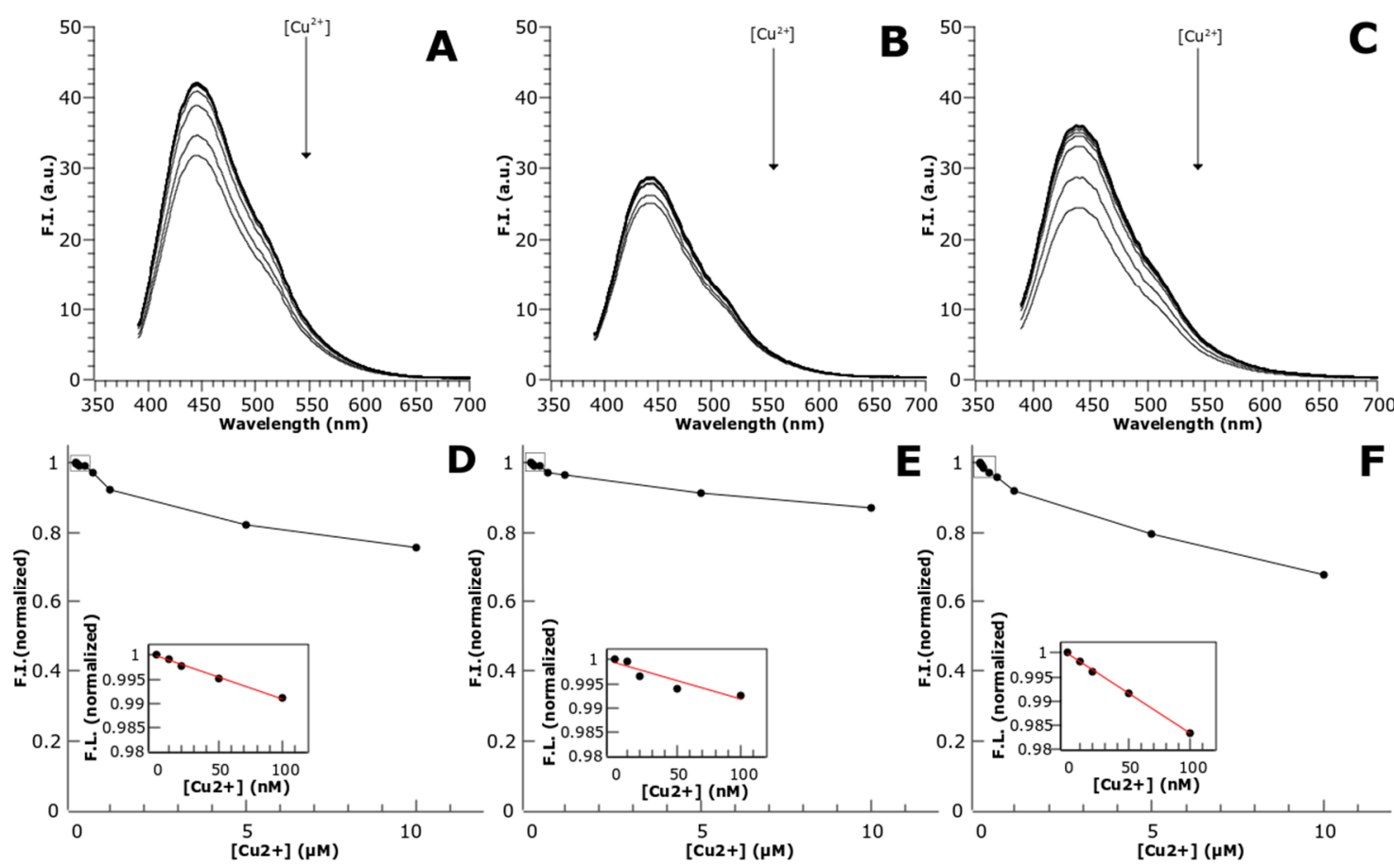

Figure 6. $\mathrm{Cu}$ detection by protein-stabilized nanoclusters. Fluorescence emission spectra of CTPR templated metal NCs in the presence of different $\mathrm{Cu}^{2+}$ concentrations (upper panels) C3_cys-CuNCs (A); C3_cys-AgNCs (B); and C3_cys-AuNCs (C). Normalized fluorescence vs. $\mathrm{Cu}^{2+}$ concentration plots (lower panels). C3_cys-CuNCs (D); C3_cys-AgNCs (E); and C3_cys-AuNCs (F).

No studies have been performed to explore the mechanism of fluorescence quenching by copper, but there are some possible explanations of this effect. The linear response of the fluorescence quenching to the $\left[\mathrm{Cu}^{2+}\right]$ for CuNCs and AuNCs, for which a $\left[\mathrm{Cu}^{2+}\right]$ of $0.1 \mu \mathrm{M}$ added to $10 \mu \mathrm{M}$ of NCs (1\% analyte relative to the sensor) results in a fluorescence decrease of approximately $1 \%$, supports a Langmuir-type adsorption behavior for the $\mathrm{Cu}^{2+}$ ions. The mechanism of quenching could be due to the paramagnetic nature of $\mathrm{Cu}^{2+}$, which can enhance intersystem crossing (ISC) as a way of non-radiative dissipation of the energy absorbed, competing with fluorescence emission, and thus quenching the fluorescence of the metal NCs [23]. Another possible explanation is that there is some de-attachment of AuNCs from the coordination sites in the protein due to a reaction with $\mathrm{Cu}^{2+}$, thus leading to a loss of fluorescence. Further characterization of this sensing behavior would be necessary in order to detangle the molecular mechanism underlying fluorescence quenching by copper.

\subsection{Reactive Oxygen Species (ROS) Detection}

The different NCs samples were evaluated as sensors for the detection of oxidative stress through the presence of reactive oxygen species in the environment. The Ag-based NCs were the only protein-templated NCs that showed reliable response to ROS (Figure 7). There was a quenching in the fluorescence of the AgNCs in response to the amount of ROS produced by RB in time. The quenching occurred slowly in time, with a $10 \%$ of fluorescence loss in an hour. It is important to note that the positive control used to detect ROS formation, ABTS, showed larger response to the presence of ROS than the AgNCs. 

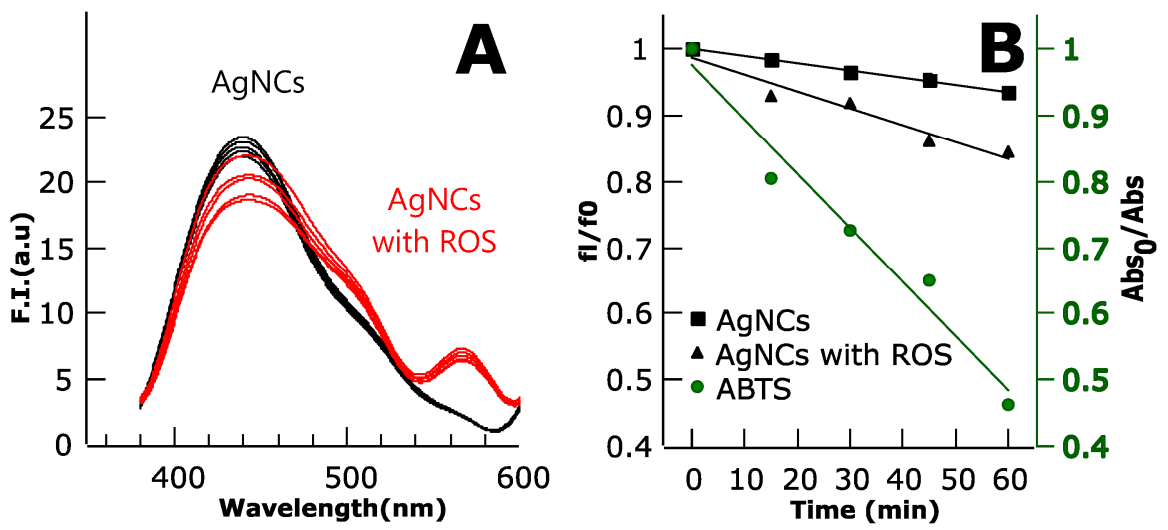

Figure 7. (A) Fluorescence emission spectra of the C3_cys AgNCs. Black lines represent no reactive oxygen species (ROS) control samples and red lines correspond to Rose Bengal-metal NCs samples, i.e., with oxidative stress from ROS production. (B) Stern-Volmer plot showing the quenching of the AgNCs fluorescence with time upon irradiation of Rose Bengal (RB), related to increased amounts of ROS. ABTS was used as a probe for ROS production; its absorbance was measured to check the formation of ROS (green spheres, absorbance in green axis).

In the case of the copper nanocluster, an initial quenching of the system before sample irradiation was observed (Figure 7A), as well as some photobleaching as seen in the control sample of the protein without ROS (Figure 7B). However, there was no further quenching upon irradiation when ROS were being synthetized (data not shown). It is possible that Rose Bengal was quenching the NCs fluorescence due to some interaction between the molecule and the Ag atoms. The mechanism on how ROS quenches the fluorescence of AgNCs was not yet solved in our system; however, it is possible that oxido-reduction changes on the AgNCs directed by ROS activity induced fluorescence quenching, as has been reported before [24,25]. In the case of the protein-templated AuNCs, the absence of response to ROS is supported by the fact that AuNCs have good redox stability, whereas in the case of the protein templated CuNCs the lack of response to ROS is not explained by their redox stability, since they are less stable than AgNCs.

\section{Conclusions}

In previous work we developed new technologies in order to use designed CTPR proteins for the controlled growth of AuNCs. The main objective of the current work was to evaluate the potential of repeat protein scaffolds as versatile templates for the synthesis and stabilization of different metal NCs (Au, Ag, and CuNCs) to explore the use of CTPR stabilized metal NCs as sensors for different conditions, such as temperature, ions, or ROS. We want to highlight the sensitivity that these NCs have for the temperature sensing and the ability to undergo several temperature cycles without losing sensing efficiency. Moreover, AuNCs can be used to detect small concentrations of copper ions in solution and AgNCs showed sensitivity to ROS species. Although there are previously reported systems that showed similar detection capabilities, and in some cases with better specificity and detection limits, the major advantage in CTPR templated metal NCs is that the same system can encode different sensing capabilities and functionalities, by just changing the metal attached. Therefore, this work demonstrates the versatility of the repeat protein scaffolds to obtain protein-stabilized nanoclusters with different metal composition, which increases their potential use in the field of biosensing. Due to the modular nature of these proteins, we envision the potential of CTPR protein templated metal NCs as integrated modules in larger assemblies of CTPR proteins. Using their modularity and with different functional elements alongside the protein array, we could achieve the building of multi-component sensing tools. 


\section{Patents}

Patent: A.A. and A.L.C. Metal Nanocluster Scaffolds. European patent (EP17382451.7), PCT application (PCT/EP2018/068710).

Author Contributions: A.A., E.L.-M. and A.L.C. conceptualized the idea; A.A. and E.L.-M. designed and carried out the experiments; A.A., E.L.-M. and A.L.C. wrote the manuscript; A.L.C. acquired the funding and administrated the project.

Funding: This work was funded by the European Research Council ERC-2014-CoG-648071-ProNANO (A.L.C.), and the Spanish Ministry of Economy and Competitiveness (MINECO) BIO2016-77367-C2-1-R (A.L.C.).

Acknowledgments: E.L.-M. thanks the Spanish Ministry of Education for the FPI grant (BIO2016-77367-C2-1-R). We acknowledge Angel Martinez for support with TEM imaging and members of the A.L.C. laboratory at CIC biomaGUNE for their comments and support.

Conflicts of Interest: The authors declare no conflict of interest. The funders had no role in the design of the study; in the collection, analyses, or interpretation of data; in the writing of the manuscript, or in the decision to publish the results.

\section{References}

1. Londoño-Larrea, P.; Vanegas, J.P.; Cuaran-Acosta, D.; Zaballos-García, E.; Pérez-Prieto, J. Water-Soluble Naked Gold Nanoclusters Are Not Luminescent. Chem. Eur. J. 2017, 23, 8137-8141. [CrossRef] [PubMed]

2. Yu, P.; Wen, X.; Toh, Y.-R.; Tang, J. Temperature-Dependent Fluorescence in $\mathrm{Au}_{10}$ Nanoclusters. J. Phys. Chem. C 2012, 116, 6567-6571. [CrossRef]

3. Huang, H.; Li, H.; Wang, A.-J.; Zhong, S.-X.; Fang, K.-M.; Feng, J.-J. Green synthesis of peptide-templated fluorescent copper nanoclusters for temperature sensing and cellular imaging. Analyst 2014, 139, 6536-6541. [CrossRef] [PubMed]

4. Chen, X.; Essner, J.B.; Baker, G.A. Exploring luminescence-based temperature sensing using protein-passivated gold nanoclusters. Nanoscale 2014, 6, 9594. [CrossRef] [PubMed]

5. Wu, Y.-T.; Shanmugam, C.; Tseng, W.-B.; Hiseh, M.-M.; Tseng, W.-L. A gold nanocluster-based fluorescent probe for simultaneous $\mathrm{pH}$ and temperature sensing and its application to cellular imaging and logic gates. Nanoscale 2016, 8, 11210-11216. [CrossRef] [PubMed]

6. Sun, Y.; Wu, J.; Wang, C.; Zhao, Y.; Lin, Q. Tunable near-infrared fluorescent gold nanoclusters: Temperature sensor and targeted bioimaging. New J. Chem. 2017, 41, 5412-5419. [CrossRef]

7. Han, B.; Hou, X.; Xiang, R.; He, G. Synthesis of highly luminescent Cu/Ag bimetal nanoclusters and their application in a temperature sensor. Anal. Methods 2017, 9, 4028-4032. [CrossRef]

8. Durgadas, C.V.; Sharma, C.P.; Sreenivasan, K. Fluorescent gold clusters as nanosensors for copper ions in live cells. Analyst 2011, 136, 933-940. [CrossRef] [PubMed]

9. Goswami, N.; Giri, A.; Bootharaju, M.S.; Xavier, P.L.; Pradeep, T.; Pal, S.K. Copper Quantum Clusters in Protein Matrix: Potential Sensor of $\mathrm{Pb}^{2+}$ Ion. Anal. Chem. 2011, 83, 9676-9680. [CrossRef] [PubMed]

10. Xie, J.; Zheng, Y.; Ying, J.Y. Highly selective and ultrasensitive detection $\mathrm{ofHg}^{2+}$ based on fluorescence quenching of $\mathrm{Au}$ nanoclusters by $\mathrm{Hg}^{2+}-\mathrm{Au}^{+}$interactions. Chem. Commun. 2010, 46, 961-963. [CrossRef] [PubMed]

11. Couleaud, P.; Adan-Bermudez, S.; Aires, A.; Mejías, S.H.; Sot, B.; Somoza, A.; Cortajarena, A.L. Designed Modular Proteins as Scaffolds to Stabilize Fluorescent Nanoclusters. Biomacromolecules 2015, 16, 3836-3844. [CrossRef] [PubMed]

12. Cano, A.; Acosta, M.; Arnao, M.B. A method to measure antioxidant activity in organic media: Application to lipophilic vitamins. Redox Rep. 2000, 5, 365-370. [CrossRef] [PubMed]

13. Wang, L.; Ding, L.; Wang, Y.; Zhang, Y.; Liu, J. Isolation and Characterisation of in Vitro and Cellular Free Radical Scavenging Peptides from Corn Peptide Fractions. Molecules 2015, 20, 3221-3237. [CrossRef] [PubMed]

14. Wang, C.; Wang, C.; Xu, L.; Cheng, H.; Lin, Q.; Zhang, C. Protein-directed synthesis of pH-responsive red fluorescent copper nanoclusters and their applications in cellular imaging and catalysis. Nanoscale 2014, 6, 1775-1781. [CrossRef] [PubMed] 
15. Kawasaki, H.; Hamaguchi, K.; Osaka, I.; Arakawa, R. ph-Dependent Synthesis of Pepsin-Mediated Gold Nanoclusters with Blue Green and Red Fluorescent Emission. Adv. Funct. Mater. 2011, 21, 3508-3515. [CrossRef]

16. Lee, I.H.; Ahn, B.; Lee, J.M.; Lee, C.S.; Jung, Y. A facile synthesis of fluorescent silver nanoclusters with human ferritin as a synthetic and interfacing ligand. Analyst 2015, 140, 3543-3550. [CrossRef] [PubMed]

17. Kuzkova, N.; Popenko, O.; Yakunov, A. Application of Temperature-Dependent Fluorescent Dyes to the Measurement of Millimeter Wave Absorption in Water Applied to Biomedical Experiments. Int. J. Biomed. Imaging 2014, 2014, 243564. [CrossRef] [PubMed]

18. Cortajarena, A.L.; Regan, L. Calorimetric study of a series of designed repeat proteins: Modular structure and modular folding. Protein Sci. Publ. Protein Soc. 2011, 20, 336-340. [CrossRef] [PubMed]

19. Kajander, T.; Cortajarena, A.L.; Main, E.R.G.; Mochrie, S.G.J.; Regan, L. A New Folding Paradigm for Repeat Proteins. J. Am. Chem. Soc. 2005, 127, 10188-10190. [CrossRef] [PubMed]

20. Cortajarena, A.L.; Mochrie, S.G.J.; Regan, L. Modulating repeat protein stability: The effect of individual helix stability on the collective behavior of the ensemble: Modulating Repeat Protein Stability. Protein Sci. 2011, 20, 1042-1047. [CrossRef] [PubMed]

21. Tian, L.; Zhao, W.; Li, L.; Tong, Y.; Peng, G.; Li, Y. Multi-talented applications for cell imaging, tumor cells recognition, patterning, staining and temperature sensing by using egg white-encapsulated gold nanoclusters. Sens. Actuators B Chem. 2017, 240, 114-124. [CrossRef]

22. Feng, J.; Chen, Y.; Han, Y.; Liu, J.; Ma, S.; Zhang, H.; Chen, X. pH-Regulated Synthesis of Trypsin-Templated Copper Nanoclusters with Blue and Yellow Fluorescent Emission. ACS Omega 2017, 2, 9109-9117. [CrossRef] [PubMed]

23. Valeur, B.; Berberan-Santos, M.N. Molecular Fluorescence: Principles and Applications; Wiley-VCH Verlag GmbH \& Co. KGaA: Weinheim, Germany, 2012; ISBN 978-3-527-65000-2.

24. Patel, A.S.; Mohanty, T. Silver nanoclusters in BSA template: A selective sensor for hydrogen peroxide. J. Mater. Sci. 2014, 49, 2136-2143. [CrossRef]

25. Zhang, L.; Liang, R.-P.; Xiao, S.-J.; Bai, J.-M.; Zheng, L.-L.; Zhan, L.; Zhao, X.-J.; Qiu, J.-D.; Huang, C.-Z. DNA-templated Ag nanoclusters as fluorescent probes for sensing and intracellular imaging of hydroxyl radicals. Talanta 2014, 118, 339-347. [CrossRef] [PubMed] 